\title{
Ultrasound findings of the urinary tract in patients with spinal cord injury: a study of 1005 cases
}

\author{
Ü Güzelküçük, Y Demir, S Kesikburun, B Aras, E Yaşar and AK Tan
}

Study design: Retrospective chart review.

Objectives: To document urinary tract abnormalities (UTAs) in patients with spinal cord injury (SCI) and to assess demographic and clinical features associated with UTA detected via ultrasound (US).

Setting: Turkish Armed Forces Rehabilitation Center, Ankara, Turkey.

Methods: The medical and radiological records of all patients with $\mathrm{SCl}$ were screened. Variables in each patient with $\mathrm{SCl}$, including age at the time of the US examination, gender, etiology, level and severity of SCI, time since injury, bladder management methods and findings of urinary tract US, were reviewed and analyzed.

Results: Data were obtained from 1005 patients during the 6-year study period (2008-2013). The mean age was 35.67 \pm 14.79 years and the male-female ratio was 2.84:1. Trabeculated bladder (TB) was observed in $35.1 \%$ of the patients, bladder calculi in $6 \%$, renal calculi in $6 \%$, hydronephrosis in $5.5 \%$ and renal atrophy in $1.2 \%$. Bladder calculi, renal calculi and renal atrophy were observed in patients with TB at higher rates than in those without TB $(P=0.001,0.036$ and 0.004 , respectively). The association of TB with hydronephrosis was very close to significance level $(P=0.052)$.

Conclusion: A large number of SCI patients had UTAs including TB, renal and bladder calculi, hydronephrosis and renal atrophy. The time since injury, level and severity of SCl and bladder management method may influence development of UTA. In addition, TB may be a helpful parameter for predicting UTA in SCl patients.

Spinal Cord (2015) 53, 139-144; doi:10.1038/sc.2014.201; published online 4 November 2014

\section{INTRODUCTION}

One of the most important sequelae after spinal cord injury (SCI) is dysfunction of the lower urinary tract (LUT) secondary to neurogenic bladder disease. Neurogenic bladder affects the functions of the LUT in different forms and degrees depending on the level and severity of SCI. LUT dysfunction can cause a variety of long-term deleterious complications including urinary tract infections, calculus disease, morphological alterations of the bladder and urethra, vesicoureteral reflux, hydronephrosis, bladder carcinoma and renal failure. ${ }^{1-5}$

The principle aim of managing LUT dysfunction is the prevention of complications and preservation of upper urinary tract function. ${ }^{1,6-8}$ Therefore, these patients need life-long urological assessment at regular intervals to minimize urinary tract-associated morbidity. ${ }^{6-8}$ Although there is no agreed protocol for the follow-up procedure, ultrasound (US) has an important role in the urinary tract surveillance of SCI patients. ${ }^{3,7}$ It is one of the most commonly used diagnostic tools in rehabilitation practice because it is widely available, noninvasive, safe, affordable and does not expose patients to any radiation. 69 US findings such as calculi, hydronephrosis and tumor can indicate other important diagnostic tools like computerized tomography scan, magnetic resonance imaging, intravenous pyelogram, cystoscopy, nuclear scans and urodynamic tests. As a policy of the Turkish Armed Forces Rehabilitation Center, US examination of the urinary tract is routinely performed for all SCI patients admitted to the hospital to detect urinary abnormalities.
The primary purpose of the present study was to document ultrasonographic findings of the urinary tract in patients with SCI. The secondary purpose was to assess demographic and clinical features associated with urinary abnormalities detected via US.

\section{MATERIALS AND METHODS}

The medical and radiological records were reviewed of all patients with SCI admitted to the Turkish Armed Forces Rehabilitation Center, a tertiary hospital, during a 6-year period (January 2008-December 2013). Patients who had not undergone urinary tract US were excluded.

Demographic and clinical data including age at the time of the US examination, gender, etiology, degree and level of neurological impairment, time since injury and bladder management methods (spontaneous/other, clean intermittent catheterization (CIC) or indwelling urethral catheter (IUC)) were recorded. The patients were divided into three groups based on time since injury: $\leqslant 1$ year, $1-5$ years and $>5$ years. The neurological level of each patient was determined according to the American Spinal Injury Association Impairment Scale (AIS).

Findings of urinary tract US were noted in each case and were analyzed separately. Trabeculated bladder (TB), renal and bladder calculi, hydronephrosis and renal atrophy, which are likely to be associated with SCI, were accepted as criteria of urinary tract abnormalities (UTAs). The number and size of the calculi, side of the abnormalities and severity of hydronephrosis were also noted. Anatomical abnormalities (for example, solitary and double kidneys, horseshoe and pelvic (ectopic) kidneys) and changes in the structure of the 
renal parenchyma (for example, kidney cyst) were also identified and documented.

The US examinations of the urinary tract were performed by a trained professional and using a sonoline Antares US scanner with $3.5 \mathrm{MHz}$ convex transducer (Siemens Medical Solutions, Erlangen, Germany). The study was approved by the Local Ethics Committee.

Statistical analysis was performed using SPSS version 15.0 (SPSS Inc., Chicago, IL, USA). Continuous variables were presented as mean \pm s.d. and range (min-max), and categorical variables were summarized as frequencies and percentages. Categorical variables were analyzed with the chi-square test. The distributions of the numerical variables were examined using the Kolmogorov-Smirnov test for normality. The differences between the groups were determined with the Mann-Whitney $U$-test. A multiple linear regression model was used to identify independent predictors (age, gender, etiology, level and severity of SCI, time since injury and bladder management method) of UTA. Statistical significance was defined as $P<0.05$.

\section{RESULTS}

A total of 1078 patients with SCI were initially enrolled into the study between January 2008 and December 2013. As US examination was not available, 73/1078 (6.8\%) patients enrolled were excluded. The demographic and clinical characteristics of patients are summarized in Table 1.

Of the 1005 eligible patients, the mean age was $35.67 \pm 14.79$ years (range: $4-82$ years) and the male-female ratio was $2.84: 1$. The mean time elapsed between the date of the injury and US examination was
$32.49 \pm 49.23$ months (range: $1-346$ months). Traumatic etiology was determined in $83 \%$ of the patients and 692 patients $(68.9 \%)$ were paraplegic. A total of 487 lesions $(48.5 \%)$ were complete, whereas 518 $(51.5 \%)$ were incomplete.

The frequency of UTAs including $\mathrm{TB}$, renal and bladder calculi, hydronephrosis and renal atrophy was 435/1005 (43.3\%) in the study population (Figure 1). Of the 1005 SCI patients, 55 (5.5\%) (mild: 41, moderate: 12 , severe: 2 ) had hydronephrosis in 76 kidneys. One (vs two)-sided hydronephrosis was more common $(61.8 \%$ vs $38.2 \%)$. Of 113 patients $(11.2 \%)$ found to have urinary calculi, $61(6.1 \%)$ had renal calculi in 73 kidneys and $61(6.1 \%)$ had bladder calculi. Nine urinary calculi were located in both locations. Renal atrophy was present in 12 patients (1.1\%). There were some significant differences between the characteristics of the patients with these abnormalities and the patients without abnormal findings as shown in Table 1. Due to renal injury occurring concurrently with SCI, unilateral nephrectomy was applied to 11 patients (1\%). Kidney cyst was observed in 45 patients, ectopic kidney in 5 patients and horseshoe kidney in 1 patient. We did not detect any tumors.

Both bladder and renal solitary stones were more common than multiple stones $(52.5 \%$ and $72.1 \%$, respectively). The mean size of bladder and renal stone was $12.44 \pm 11.82 \mathrm{~mm}$ (range: $1-37 \mathrm{~mm}$ ) and $6.82 \pm 4.73 \mathrm{~mm}(1-28 \mathrm{~mm})$, respectively, and 43 (70.5\%) bladder stones were $>1.0 \mathrm{~cm}$. The size distribution of renal stones was as follows: $1-5 \mathrm{~mm}$ : 52.5\%; 6-10 mm: 32.8\%; > $10 \mathrm{~mm}$ : $14.8 \%$.

Table 1 Characteristics of patients with and without urinary tract abnormalities

\begin{tabular}{|c|c|c|c|c|c|c|c|}
\hline & $\begin{array}{l}\text { All subjects } \\
(\mathrm{n}=1005)\end{array}$ & $\begin{array}{l}\text { Without abnormal } \\
\text { findings }(n=569)\end{array}$ & $\begin{array}{c}\text { Trabeculated } \\
\text { bladder }(\mathrm{n}=353)\end{array}$ & $\begin{array}{l}\text { Bladder calculi } \\
\qquad(\mathrm{n}=61)\end{array}$ & $\begin{array}{l}\text { Renal calculi } \\
\qquad(\mathrm{n}=61)\end{array}$ & $\begin{array}{l}\text { Hydronephrosis } \\
(\mathrm{n}=55)\end{array}$ & $\begin{array}{l}\text { Renal atrophy } \\
(\mathrm{n}=12)\end{array}$ \\
\hline Age (years) mean \pm s.d. & $35.67 \pm 14.79$ & $36.17 \pm 14.35$ & $34.39 \pm 15.28 *$ & $29.61 \pm 11.95^{*}$ & $32.28 \pm 17.93$ & $36.09 \pm 16.07$ & $39.00 \pm 14.69$ \\
\hline \multicolumn{8}{|l|}{ Gender, n (\%) } \\
\hline Male & $743(73.9)$ & $403(70.8)$ & $277(78.4)^{*}$ & $51(83.6)^{*}$ & $52(85.2)^{*}$ & $39(70.9)$ & $12(100)^{*}$ \\
\hline Female & $262(26.2)$ & $166(29.2)$ & $76(21.5)$ & $10(16.4)$ & $9(14.8)$ & $16(29.1)$ & 0 \\
\hline Mean time since injury (months) \pm s.d. & $32.49 \pm 49.23$ & $28.76 \pm 43.76$ & $38.99 \pm 54.28^{*}$ & $14.03 \pm 12.97$ & $32.08 \pm 52.15$ & $63.44 \pm 92.53^{*}$ & $91.92 \pm 117.62^{*}$ \\
\hline \multicolumn{8}{|l|}{ Duration of SCl, n (\%) } \\
\hline$\leqslant 1$ year & $459(45.7)$ & $288(50.6)$ & $125(35.4)^{*}$ & $35(57.4)^{*}$ & $29(47.5)$ & $17(30.9)^{*}$ & $3(25)$ \\
\hline $1-5$ years & $406(40.4)$ & $210(36.9)$ & $170(48.2)$ & $26(42.6)$ & $23(37.7)$ & $23(41.8)$ & $5(41.7)$ \\
\hline$>5$ years & $140(13.9)$ & $71(12.5)$ & $58(16.4)$ & 0 & $9(14.8)$ & $15(27.3)$ & $4(33.3)$ \\
\hline \multicolumn{8}{|l|}{ Mechanism of injury, n (\%) } \\
\hline Traumatic & $834(83)$ & $467(82.1)$ & $298(84.4)$ & $59(96.7)^{*}$ & $45(73.8)$ & $47(85.5)$ & $10(83.3)$ \\
\hline Non-traumatic & $171(17)$ & $102(17.9)$ & $55(15.6)$ & $2(3.3)$ & $16(26.2)$ & $8(14.5)$ & $2(16.7)$ \\
\hline \multicolumn{8}{|l|}{ Level of SCl, n (\%) } \\
\hline Tetraplegia & $313(31.1)$ & $154(27.1)$ & $136(38.5)^{*}$ & $26(42.6)^{*}$ & $26(42.6)^{*}$ & $18(32.7)$ & $4(33.3)$ \\
\hline Paraplegia & $692(68.9)$ & 415 (72.9) & $217(78.5)$ & $35(57.4)$ & $35(57.4)$ & $37(67.3)$ & $8(66.7)$ \\
\hline \multicolumn{8}{|l|}{ Severity of injury, n (\%) } \\
\hline Incomplete & $518(51.5)$ & $322(51.1)$ & $151(42.8)^{*}$ & $29(47.5)$ & $35(57.4)$ & $25(45.5)$ & $7(58.3)$ \\
\hline Complete & $487(48.5)$ & $247(43.4)$ & $202(57.2)$ & $32(52.5)$ & $26(42.6)$ & $30(54.5)$ & $5(41.7)$ \\
\hline \multicolumn{8}{|l|}{ Bladder management methods, $\mathrm{n}(\%)$} \\
\hline Spontaneous/other & $236(23.5)$ & $174(30.6)$ & $44(12.5)^{*}$ & $9(14.8)^{*}$ & $16(26.2)$ & $10(18.2)^{*}$ & $2(16.7)$ \\
\hline $\mathrm{ClC}$ & $607(60.4)$ & $318(55.9)$ & $245(69.4)$ & $37(60.7)$ & $33(54.1)$ & $27(49.1)$ & $8(66.7)$ \\
\hline Indwelling catheter & $162(16.1)$ & $77(13.5)$ & $64(18.1)$ & $15(24.6)$ & $12(19.7)$ & $18(32.7)$ & $2(16.7)$ \\
\hline
\end{tabular}

Abbreviations: $\mathrm{CIC}$, clean intermittent catheterization; $\mathrm{SCl}$, spinal cord injury.

${ }^{*} P<0.05$ vs without abnormal findings. 
TB was detected in $35.1 \%$ patients. Of the patients with TB, 9.3\% had bladder calculi, $8.2 \%$ had renal calculi, $7.4 \%$ had hydronephrosis and $2.5 \%$ renal atrophy. Bladder calculi, renal calculi and renal atrophy were observed in patients with $\mathrm{TB}$ at higher rates than in those without TB ( $P=0.001,0.036$ and 0.004 , respectively) (Table 2). Statistically significant differences between the patients with TB and those without abnormal findings were noted for gender, mean time since injury, duration, level and severity of SCI and bladder management method $(P<0.05)$.

In patients with paraplegia and tetraplegia, UTA was determined at $40 \%$ and $50.8 \%$, respectively. TB, bladder and renal calculi were more common among patients with tetraplegia than those with paraplegia ( $P=0.0001,0.046$ and 0.046 , respectively). The percentage of UTA in patients with incomplete and complete injury was $37.8 \%$ and $49.3 \%$, respectively. TB was more common in complete injury $(P=0.0001)$ (Table 3 ). The observed UTA rates were $37.2 \%$ for $\leqslant 1$ year, $48.3 \%$ for

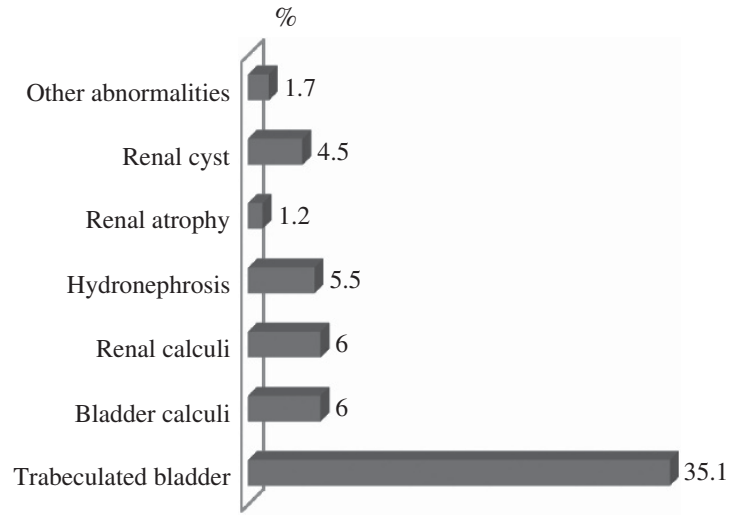

Figure 1 The distribution of urinary tract findings in patients with $\mathrm{SCl}$.

Table 2 Abnormalities of the urinary tract according to the presence or absence of trabeculated bladder

\begin{tabular}{lccc}
\hline & $\begin{array}{c}\text { Without trabeculated } \\
\text { bladder } \\
(\mathrm{n}=652), \mathrm{n}(\%)\end{array}$ & $\begin{array}{c}\text { With trabeculated } \\
\text { bladder }\end{array}$ & \\
& $28(4.3)$ & $33(9.3)$ & 0.001 \\
Bladder calculi & $32(4.9)$ & $29(8.2)$ & 0.036 \\
Renal calculi & $29(4.4)$ & $26(7.4)$ & 0.052 \\
Hydronephrosis & $3(0.5)$ & $9(2.5)$ & 0.004 \\
Renal atrophy & $(\mathrm{n}=353)$ & P-value \\
\hline
\end{tabular}

Values in bold indicate statistically significant differences at 5 percent.
$1-5$ years and $49.3 \%$ for $>5$ years after injury. There were significant differences between the time since injury groups with regard to TB, bladder calculi and hydronephrosis $(P<0.05)$ (Figure 2).

Bladder management methods were spontaneous/other in 236 $(23.5 \%)$ of the patients, CIC in $607(60.4 \%)$ and IUC in 162 $(16.1 \%)$. There were significant differences among the participants in the three bladder management methods with respect to percentages of TB and hydronephrosis $(P<0.05)$ (Figure 3 ).

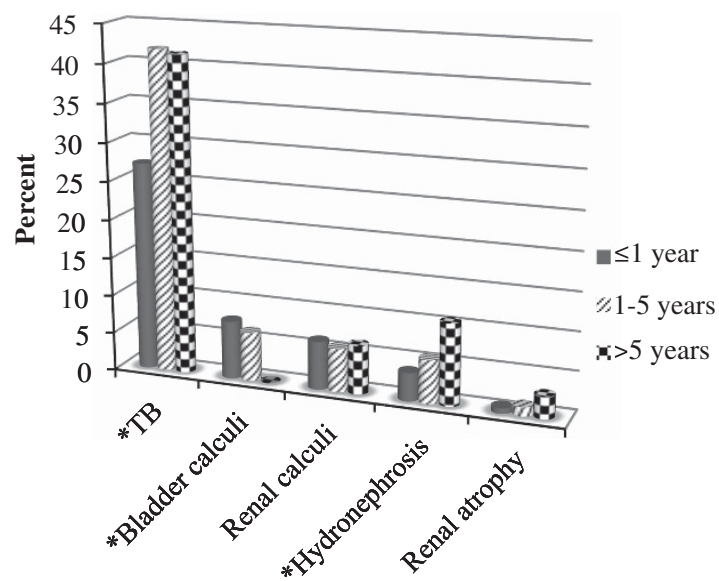

Figure 2 Incidence of the complications according to the time since injury. $* P<0.05$

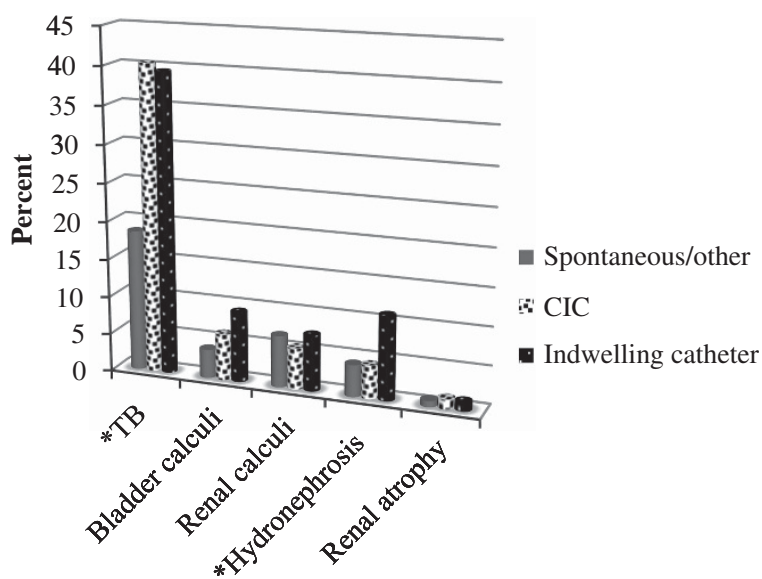

Figure 3 Incidence of the complications according to bladder management methods. ${ }^{*} P<0.05$

Table 3 Urinary tract abnormalities according to the level and severity of the injury

\begin{tabular}{|c|c|c|c|c|c|c|}
\hline Trabeculated bladder & $136(43.5)$ & 217 (31.4) & 0.0001 & $202(41.5)$ & $151(29.2)$ & 0.0001 \\
\hline Renal calculi & $26(8.3)$ & $35(5.1)$ & 0.046 & $26(5.3)$ & $35(6.8)$ & 0.347 \\
\hline Hydronephrosis & $18(5.8)$ & $37(5.3)$ & 0.794 & $30(6.2)$ & $25(4.8)$ & 0.353 \\
\hline Renal atrophy & $4(1.3)$ & $8(1.2)$ & 0.869 & $5(1)$ & $7(1.4)$ & 0.636 \\
\hline
\end{tabular}

Values in bold indicate statistically significant differences at 5 percent. 
A male predominance was seen in $\mathrm{TB}$, renal calculi and renal atrophy cases $(P=0.016,0.038$ and 0.039 , respectively). There were statistically significant differences between the male and female patients with respect to the level and severity of SCI and bladder management methods $(P<0.05)$. Tetraplegia, complete lesion, CIC and IUC were more common in male patients than female ones (34.6\% vs $21.4 \%, 51.5 \%$ vs $39.7 \%, 63.3 \%$ vs $52.3 \%$ and $16.3 \%$ vs $15.6 \%$, respectively).

The variables that strongly predicted the occurence of UTA based on logistic regression analysis are presented in Table 4.

\section{DISCUSSION}

Despite advances in rehabilitative care and treatment of SCI, complications of the urinary tract are still a major problem in long-term management. Therefore, early detection and appropriate treatment of possible further complications is important to prevent upper urinary tract deterioration, which is associated with increased morbidity, and premature mortality. ${ }^{1-3}$

It is well known that patients with SCI have an increased risk of urinary calculi compared with the general population with a higher chance of recurrence. ${ }^{5,10-12}$ Calculi formation is likely multifactorial with multiple lithogenic factors. Risk factors in patients with SCI are as follows: recurrent urinary tract infections, high intravesical pressure and subsequent vesicoureteric reflux, hypercalciuria secondary to reduced mobility, metabolic disturbances and environmental factors. $5,10,11,13,14$ Urinary calculi in SCI patients is associated with significant morbidity, such as recurrent urinary tract infection and loss of renal function. $3,7,10,13$

Chen et al. ${ }^{14,15}$ found a decreasing trend over the past few decades in bladder calculi, but not in renal calculi in SCI cases. A wide range of incidence of urinary calculi in SCI patients has been previously reported, with incidences of bladder and renal calculi ranging from 1.3 to $28^{3,7,8,10,12,15}$ and 3.3 to $28,{ }^{5,12,14}$ respectively. This discrepancy might be due to differences in follow-up period and patient characteristics, including age, gender, etiology, injury level and severity, time since injury and bladder management methods. The incidence of urinary calculi in the current study population, which was detected in both bladder (6\%) and kidney (6\%) equally, was $11.2 \%$. SCI patients have a high incidence of bilateral renal calculi, which has been reported between 25 and $75 \% .{ }^{10}$ However, the current study population had lower rates of bilateral renal calculi (19.7\%). It should be noted that the sensitivity of US for diagnosing small renal calculi varies from 24 to $96 \%$, which is a less sensitive investigation than computerized tomography scan. ${ }^{9}$ If computerized tomography scan had been used, a greater incidence of renal calculi might be seen.

One of the factors which may have a negative impact on developing UTA is the bladder management method. CIC was the most common method of bladder management among the patients of this study. It is clear that CIC is a safer bladder management method than IUC. The bladder management method has been reported to have no effect on renal calculi formation. ${ }^{8,15}$ Bartel et al. ${ }^{5}$ noted bladder calculi by IUC in $6.6 \%$ and by $\mathrm{CIC}$ in $2 \%$. Chen et al. ${ }^{14}$ found that IUC was associated with a substantially greater risk of bladder calculi; however, $\mathrm{Ku}$ et al. ${ }^{12}$ did not show this relationship with bladder calculi. In the present study, bladder calculi was observed 3.8\% in spontaneous/other, $6.1 \%$ in CIC and $9.3 \%$ in IUC, although renal calculi had similar incidence rates among bladder management methods. These results suggest that there is a stronger relationship between bladder management methods and calculi formation in the bladder than in the kidney.

Another important factor which has an influence on bladder calculi formation is the time since injury. ${ }^{5,12}$ Chen et al. ${ }^{14,15}$ reported that the 
risk for bladder and renal calculi reached its peak during the first 3 months after SCI and decreased after the first year. In the current study, it was observed that the highest occurence of bladder calculi was during the first year after SCI. However, renal calculi was determined at similar incidence rates in the three groups based on time since injury. Patients with duration of injury of $1-5$ years or $>5$ years might have already had renal calculi in the first year of SCI, but that information was not available.

The literature includes inconsistent findings concerning the effect of level and severity of SCI on urinary calculi formation, which complete and high level injuries have been found associated with urinary calculi in some studies. ${ }^{10-12}$ In the present study, urinary calculi were more frequent in patients with tetraplegia than those with paraplegia. But, these associations remained non-significant in the logistic regression analysis. This result may be due to a higher percentage of IUC use in patients with tetraplegia than those with paraplegia (20.1\% vs $14.3 \%)$. In addition, the severity of injury was an insignificant factor in predicting urinary calculi formation.

Patients with SCI are also prone to the development of hydronephrosis. The renal pelvis and calyceal system may be dilated as a result of high pressure, a poorly compliant bladder, calculus disease and detrusor sphincter dysynergia in SCI patients. ${ }^{4}$ Early diagnosis is important because hydronephrosis can lead to renal insufficiency or kidney damage especially if it is obvious and left untreated. ${ }^{7,16}$ No case of hydronephrosis was reported by Sipski et al. ${ }^{2}$ In the present study, the incidence of hydronephrosis was $5.4 \%$, which is consistent with the finding of Tins et al. ${ }^{3}(5 \%)$ and Edokpolo et al. ${ }^{7}$ (6\%). The majority of cases were of mild hydronephrosis (74.5\%). In this study, the time since injury and use of IUC were two variables associated with the development of hydronephrosis. It is not surprising that patients of over 5 years after SCI had the highest proportion of hydronephrosis. Interestingly, hydronephrosis was 2.5 -fold higher in patients with IUC than in those with CIC. One of the reasons of such a confounding finding might have been that IUC had been used for the management of hydronephrosis that had been already diagnosed.

High detrusor pressure leads to potential structural deterioration of the bladder wall, causing TB as a result of a bladder outlet obstruction and neurogenic bladder. ${ }^{1,6,17}$ Hoffberg and Cardenas ${ }^{17}$ reported that $\mathrm{TB}$ at $57 \%$ overall and in $31 \%$ of patients within 12 months of SCI. In this study, the most common UTA was TB (35.1\%) and the incidence was $27.2 \%$ in patients within the first year after SCI. Logistic regression analysis demonstrated that $\mathrm{TB}$ was significantly associated with time since injury, tetraplegia, completeness, CIC and IUC. Patients with TB were more likely to have bladder and renal calculi and renal atrophy. The association of $\mathrm{TB}$ with hydronephrosis was very close to significance level $(P=0.052)$. According to these findings, SCI patients with TB are at an increased risk of UTA. Therefore, it can be strongly recommend that SCI patients with TB detected via USG should be monitored more closely because the occurrence of UTA can be predicted with the presence of TB.

Although there are different suggestions regarding the necessity and duration of close monitoring via US in literature, ${ }^{2,7}$ we recommend annual follow-up with US examination for all our patients with SCI, regardless of whether they have urinary symptoms or not, after the first few years post injury. Not only is UTA a frequent finding but many of these abnormalities are silent in SCI patients. ${ }^{3,6}$ The majority of UTA is detected as an incidental finding on US. Therefore, evaluation of the urinary tract with regular follow-up should be applied to avoid missing urinary abnormalities in these high-risk patients. High rate of UTA found in the present study supports this.
To the best of our knowledge, this study reports the largest series of ultrasonographic findings of both upper and lower urinary tract in patients with SCI. In addition, we think that the present findings provide a comprehensive picture of Turkish SCI patients, both because the Turkish Armed Forces Rehabilitation Center treats SCI patients from all regions of Turkey, and because the majority of patients (93.2\%) underwent urinary tract US. Furthermore, the fact that all ultrasounds were performed by a single radiologist who was specially trained in urinary tract US is another strength of this study. However, this study has several limitations including its retrospective and singlecenter design. Second, urinary symptoms, renal function (creatinine), urodynamic findings, metabolic abnormalities, duration of bladder management methods and use of anticholinergic medication were not evaluated. Finally, there was no information available as to the exact time of occurrence of UTA and whether the urinary calculi was firsttime or recurrent calculi.

The present study, which mainly focused on the incidence and risk factors of UTA, provides important information on the epidemiology of UTA in patients with SCI. This study indicates that a large number of SCI patients have UTA detected via US and there is a requirement for continuous assessment in order to minimize morbidity. According to the results of this study, the time since injury, level and severity of SCI and bladder management method were associated with a substantially increased risk of UTA. TB indicated a high risk of developing UTA, and it may be helpful parameter for predicting UTA in SCI patients.

\section{DATA ARCHIVING}

There were no data to deposit.

\section{CONFLICT OF INTEREST}

The authors declare no conflict of interest.

\section{ACKNOWLEDGEMENTS}

We are grateful to Dr Serkan Ilkbahar, Department of Radiology, Turkish Armed Forces Rehabilitation Center, Ankara, Turkey for his valuable guidance and support in completing this work. It has not been previously published in any form of presentation.

1 Silva JA, Gonsalves MD, de Melo RT, Carrerette FB, Damião R. Association between the bladder wall thickness and urodynamic findings in patients with spinal cord injury. World J Urol (e-pub ahead of print 27 February 2014).

2 Sipski ML, Estores IM, Alexander CJ, Guo X, Chandralapaty SK. Lack of justification for routine abdominal ultrasonography in patients with chronic spinal cord injury. J Rehabil Res Dev 2004; 41: 101-108.

3 Tins B, Teo HG, Popuri R, Cassar-Pullicino V, Tyrrell P. Follow-up imaging of the urinary tract in spinal injury patients: is a KUB necessary with every ultrasound? Spinal Cord 2005; 43: 219-222.

4 Gormley EA. Urologic complications of the neurogenic bladder. Urol Clin North Am 2010; 37: 601-607.

5 Bartel P, Krebs J, Wöllner J, Göcking K, Pannek J. Bladder stones in patients with spinal cord injury: a long-term study. Spinal Cord 2014; 52: 295-297.

6 Pannek J, Bartel P, Göcking K, Frotzler A. Clinical usefulness of ultrasound assessment of detrusor wall thickness in patients with neurogenic lower urinary tract dysfunction due to spinal cord injury: urodynamics made easy? World J Urol 2013; 31: 659-664.

7 Edokpolo LU, Foster HE Jr. Renal tract ultrasonography for routine surveillance in spinal cord injury patients. Top Spinal Cord Inj Rehabil 2013; 19: 54-60.

$8 \mathrm{Ku} \mathrm{JH}$, Choi WJ, Lee KY, Jung TY, Lee JK, Park WH et al. Complications of the upper urinary tract in patients with spinal cord injury: a long-term follow-up study. Urol Res 2005; 33: 435-439.

9 Durr-E-Sabih, Khan AN, Craig M, Worrall JA. Sonographic mimics of renal calculi. J Ultrasound Med 2004; 23: 1361-1367.

10 Welk B, Fuller A, Razvi H, Denstedt J. Renal stone disease in spinal-cord-injured patients. J Endourol 2012; 26: 954-959.

11 Ost MC, Lee BR. Urolithiasis in patients with spinal cord injuries: risk factors, management, and outcomes. Curr Opin Urol 2006; 16: 93-99. 
12 Ku JH, Jung TY, Lee JK, Park WH, Shim HB. Risk factors for urinary stone formation in men with spinal cord injury: a 17-year follow-up study. BJU Int 2006; 97: 790-793.

13 Veenboer PW, Ruud Bosch JL, van Asbeck FW, de Kort LM. Urolithiasis in adult spina bifida patients: study in 260 patients and discussion of the literature. Int Urol Nephrol 2013; 45: 695-702.

14 Chen Y, DeVivo MJ, Lloyd LK. Bladder stone incidence in persons with spinal cord injury: determinants and trends, 1973-1996. Urology 2001; 58: 665-670.
15 Chen Y, DeVivo MJ, Roseman JM. Current trend and risk factors for kidney stones in persons with spinal cord injury: a longitudinal study. Spinal Cord 2000; 38: 346-353.

16 Tseng FF, Huang YH, Chen SL, Tsai SJ, Ho CC, Bih LI. Value of Doppler ultrasonography in predicting deteriorating renal function after spinal cord injury. Radiol Med 2012; 117: 500-506.

17 Hoffberg HJ, Cardenas DD. Bladder trabeculation in spinal cord injury. Arch Phys Med Rehabil1986; 67: 750-753. 